Pacific

Journal of

Mathematics

NOTES ON TANGLES, 2-HANDLE ADDITIONS AND EXCEPTIONAL DEHN FILLINGS

W. Menasco And X. Zhang 


\title{
NOTES ON TANGLES, 2-HANDLE ADDITIONS AND EXCEPTIONAL DEHN FILLINGS
}

\author{
W. Menasco and X. Zhang
}

\begin{abstract}
This paper is a set of notes concerning the following related topics in 3-manifold topology: $n$-strand tangles, handle additions, the cabling conjecture, and exceptional Dehn fillings.
\end{abstract}

\section{Introduction.}

In this paper we consider several related problems in 3-manifold topology. Here is a brief description of the content and organization of the paper. Definitions of terms which occur can be found in relevant sections.

Classical tangles, i.e., tangles in a 3-ball, have been heavily studied. Here we consider tangles in a general compact 3-manifold with boundary a 2sphere. In Section 2 we give a complete classification of the set of irreducible, non-split, completely tubing compressible tangles with two strands. To give a complete description of such tangles with more than two strands in terms of the mutual positions of the strands seems to be a difficult problem. A conjecture is raised, which suggests possible pictures (classification) of such tangles. Our study indicates that certain conditions posed on the complement of a tangle determines the tangle itself, up to topological equivalence.

The ambient space of a tangle can be considered as a union of the exterior of the tangle and several 2-handles. Therefore, problems about tangles are closely connected to problems about 2-handle additions, in particular to problems about Dehn fillings. Through such a connection, we produce, in Section 3, classes of hyperbolic knots in solid torus such that their exteriors admit Dehn filling along their outside boundary torus producing manifolds which are solid torus or Seifert fibered spaces. In the same section, we also show that if a surgery on a hyperbolic knot in a solid torus produces a Seifert fibered space, then the surgery slope, with respect to the standard meridian-longitude coordinates of the knot, must be an integer slope.

One of our motivations of studying general tangles here is our concern about the cabling conjecture. Recall that the cabling conjecture asserts that if a knot in the 3 -sphere is not a cabled knot (which includes the trivial knot), then it does not admit any surgery resulting a reducible manifold [GS]. This conjecture has been proven true for satellite knots $[\mathbf{S c h}]$, strongly invertible knots $[\mathbf{E}]$, alternating knots $[\mathbf{M T}]$, arborescent knots $[\mathbf{W}]$, symmetric knots $[\mathbf{H S}],[\mathbf{G L 4}]$ and genus one knots $[\mathbf{B Z}]$. It is also known that if a surgery 
on a non-trivial knot in $S^{3}$ produces a reducible manifold, then the surgery slope is not $0[\mathbf{G a}]$, in fact it must be an integer [GL2] with absolute value larger than one [GL3], and the reducible manifold contains a lens space summand [GL3]. In Section 4, we look at the conjecture from the point of view of tangle sums. Namely, if some surgery on a knot in $S^{3}$ produces a reducible manifold, then a reducing 2 -sphere will decompose the resulting manifold into a union of two tangles whose strands come from the core of the filling solid torus. We give some partial results along this line. Notably, we show that such tangle decomposition cannot give summands which are 2-strand tangles.

Our investigation of the cabling conjecture is extended to Section 5 but restricted to the class of knots in $S^{3}$ whose exteriors do not contain meridionally-incompressible closed essential surfaces. Note that this class of knots include all alternating knots $[\mathbf{M}]$, almost alternating knots, toroidally alternating knots and Montesinos knots $[\mathbf{A}]$. We show that if such knot exterior admits a filling yielding a reducible manifold, then the reducible manifold must be a connected sum of two lens spaces. We also show that such knot exterior does not admit any Dehn filling producing a large Seifert fibered space. It is a conjecture that every knot in $S^{3}$ does not admit a surgery yielding a large Seifert fibered 3-manifold. This conjecture can be considered as an extension of the cabling conjecture, in some sense.

Studying exceptional Dehn surgery (filling) on hyperbolic knot (exterior), i.e., surgery (filling) which produces non-hyperbolic manifolds, is a basic subject in 3-manifold topology. Most of the problems and results described above belong to this subject. Given a knot $K$ in a compact orientable 3manifolds $W$ with non-empty boundary such that the exterior of $K$ in $W$ is a simple manifold, an exceptional surgery on $W$ along $K$ will produce a manifold which contains either a reducing 2-sphere, or essential annulus, or $\partial$-reducing disk or essential torus. Sharp upper bounds on the distances (i.e., the geometric intersection numbers) between all these types of exceptional surgery slopes have been found. (See [GW, Introduction] for a table summary of these bounds.) But if one singles out Seifert Dehn surgery-surgery which yields a Seifert fibered space- as a special type of exceptional surgery, then it is still unknown what are the optimal upper bounds on the distances between a Seifert Dehn surgery slope and other types of exceptional surgery slopes. There are 5 bounds to be determined. In Section 6, the last section of the paper, we resolve this issue in four of the total five cases. We show that if one surgery on $W$ along $K$ produces a Seifert surgery and another surgery on $W$ along $K$ produces a reducible or $\partial$-reducible manifold, then the distance between the two surgery slopes is at most one. Previous known bounds in both cases were 2, obtained in $[\mathbf{W} 2]$ and $[\mathbf{G W}]$ respectively. The new bound one is optimal as it can be realized by infinitely many examples found in $[\mathbf{E W}$, Section 2]. We also show that if one surgery on $W$ along 
$K$ produces a Seifert surgery and another surgery on $W$ along $K$ produces a manifold which contains an essential torus or essential annulus, then the distance between the two surgery slopes is at most three. This bound is also sharp; a family of infinitely many examples which realize the bound will be given in Section 6.

Various techniques and results, mainly coming from [CGLS], [D], [GL3], $[\mathbf{W}],[\mathbf{B Z 2}],[\mathbf{G L}],[\mathbf{G W 2}]$, will be applied. We refer to $[\mathbf{H}],[\mathbf{J}],[\mathbf{R}]$ for standard terminology in 3-manifold topology and knot theory. Throughout the piecewise linear category is assumed for manifolds and their maps.

\section{Tangles.}

Let $W$ be a connected compact orientable 3-manifold whose boundary is a 2sphere. A tangle of $k$ strands in $W$ is a set of $k \geq 1$ mutually disjoint simple arcs properly embedded in $W$. We shall use $\left(W ; \alpha_{1}, \alpha_{2}, \ldots, \alpha_{k}\right)$ to denote a tangle in $W$ with $k$ strands $\alpha_{i}, 1 \leq i \leq k$. Two tangles $\left(W ; \alpha_{1}, \ldots, \alpha_{k}\right)$ and $\left(W^{\prime} ; \alpha_{1}^{\prime}, \ldots, \alpha_{k}^{\prime}\right)$ are considered the same (topologically equivalent) if there is a homeomorphism $h: W \rightarrow W^{\prime}$ such that $h\left(\alpha_{i}\right)=\alpha_{i}^{\prime}$ for each of $1 \leq i \leq k$. For a tangle $\left(W ; \alpha_{1}, \ldots, \alpha_{k}\right)$, we shall always let $H_{i}$ denote a regular neighborhood of $\alpha_{i}$ in $W$ such that $H_{1}, \ldots, H_{k}$ are mutually disjoint, $A_{i}=\overline{\partial H_{i}-\partial W}, c_{i}$ the center circle of $A_{i}, P=\overline{\partial W-\left(H_{1} \cup \ldots \cup H_{k}\right)}$ and $X=\overline{W-\left(H_{1} \cup \ldots \cup H_{k}\right)}$. Naturally $H_{1}, \ldots, H_{k}$ can be considered as 2handles, which when attached to $X$ along $A_{i}$ 's give the manifold $W$. A tangle $\left(W ; \alpha_{1}, \alpha_{2}, \ldots, \alpha_{k}\right)$ is called reducible if and only if $X$ is a reducible 3 -manifold, called split if and only if $P$ is compressible in $X$, called toroidal if and only if $X$ contains an incompressible torus and called annular if and only if $X$ contains a properly embedded annulus whose boundary lies in $P$ but is not isotopic in $(X, P)$ to any of $A_{i}$. Obviously the study of reducible or split tangles can be reduced to that of irreducible and non-split ones.

Let $\left(W ; \alpha_{1}, \ldots, \alpha_{k}\right)$ be an irreducible non-split tangle. Then by definition, $P$ is an incompressible planar surface in $X$ which is irreducible. Let $F_{i}$ be the surface $P \cup A_{i}, i=1, \ldots, k$. We call $F_{i}$ the surface obtained from $P$ by tubing along $A_{i}$. Then $F_{i}$ is a punctured torus (when $k>1$ ) with two less boundary components than $P$ or a closed torus (when $k=1$ ) embedded in $X$, but $F_{i}$ may or may not be incompressible in $X$. If $F_{i}$ is incompressible, we call the surface $P A_{i}$-tubing incompressible. If $F_{i}$ is compressible in $X$, we perform compressing operations on $F_{i}$ as much as possible. Then eventually we either get an incompressible surface embedded in $X$ which has less boundary components than $P$ but is not isotopic to any of $A_{j}$ or every component of the surface resulting from compressing $F_{i}$ is isotopic to some of the annuli $A_{j}$. In the latter case, we call $P$ completely $A_{i}$ tubing compressible. If $P$ is completely $A_{i}$-tubing compressible for each of $i=1, \ldots, k$, then we call such tangle completely tubing compressible. The 
following lemma gives a characterization of the ambient spaces of irreducible non-split tangles which are completely tubing compressible.

Lemma 1. If $\left(W ; \alpha_{1}, \ldots, \alpha_{k}\right)$ is an irreducible non-split completely tubing compressible tangle, then either $W$ is a punctured lens space or $W$ is a 3-ball, $k=1$ and $\alpha_{1}$ is a trivial strand in $W$.

A lens space in this paper is always assumed to be non-trivial, i.e., it is neither $S^{3}$ nor $S^{2} \times S^{1}$. We use the standard notation $L(p, q)$ to denote the lens space whose Heegaard diagram is a $(q, p)$ curve on the boundary of a solid torus $V=D^{2} \times S^{1}$ where $q$ is the meridian coordinate of the curve and $p$ is the longitude coordinate with respect to a fixed longitude of $V$. So under this convention, we have $\pi_{1}(L(p, q))=\mathbb{Z}_{|p|}$ with $1<|p|<\infty$ and with $(p, q)=1$. By a punctured lens space $L^{0}(p, q)$, we mean the complement of an open 3 -ball in $L(p, q)$. It is well known that $L^{0}(p, q)$ is well defined, i.e., is independent of the choice of the 3-ball.

The proof of Lemma 1 is essentially contained in [CGLS]. For the convenience of the reader, we give a sketch of proof in our current setting. We also need some more properties about such tangles (Corollary 2 below) obtained during the proof.

Proof. Note that $P$ is incompressible, $X$ is irreducible, $\partial X$ has genus $k$ and $F_{i}$ is a punctured torus with $2(k-1)$ boundary components. Consider the maximal compression body $X_{i}$ in $X$ based on the surface $F_{i}$; i.e., $X_{i}$ is a regular neighborhood in $X$ of the union of $F_{i}$ and all possible compressing disks for $F_{i}$ in $X$, with all possible 2 -sphere boundary components capped off by 3 -balls. The frontier of $X_{i}$, i.e., $\overline{\partial X_{i}-F_{i}}$, is a set (possibly empty) of disjoint embedded surfaces each of which must be incompressible in $X$. Since $F_{i}$ is completely compressible, each component of $\overline{\partial X_{i}-F_{i}}$ is isotopic to one of the annuli $A_{j}$. Therefore $X_{i}$ is really the $X$ minus some regular neighborhood of all $A_{j}, j \neq i$, in $X$. Hence in particular $X$ is a handlebody of genus $k$.

If $k=1$, then $X=X_{1}$ is a solid torus and $W$ is the union of $X$ and the 2-handle $H_{1}$. Hence $W$ is a lens space unless $P$ is isotopic to $A_{1}$ in which case $W$ is a 3 -ball and $\alpha_{1}$ is a trivial (boundary parallel) arc in $W$. Hence we may assume that $k>1$.

The same argument as that of [CGLS, Lemma 2.1.2] shows that there exist mutually disjoint (properly embedded) disks $E_{i}^{j}$ in $X, j \neq i$, such that $E_{i}^{j}$ meets $c_{j}$, the center circle of $A_{j}$, transversely in a single point and is disjoint from $c_{m}$ if $m \neq i$ or $j$. We remark that $E_{i}^{j}$ must intersect $c_{i}$ as otherwise it would imply that $P$ be compressible. As $W$ is obtained from $X$ by attaching 2-handles $H_{j}$ along $A_{j}$, the existence of the disks $E_{i}^{j}$ implies that attaching any collection of 2-handles $H_{j}$ to $X$ along $A_{j}, j \neq i$, always produces a handlebody. In particular, we have: 
Corollary 2. Let $\left(W ; \alpha_{1}, \ldots, \alpha_{k}\right)$ be an irreducible non-split tangle which is completely tubing compressible. Then $\overline{W-H_{i}}$ is a solid torus for each of $i$.

We continue the proof of Lemma 1 . We know now that for any $i$ and $j$, $\overline{W-\left(H_{i} \cup H_{j}\right)}$ is a handlebody of genus two. Also as in [CGLS, Lemma 2.1.4] one can show that the planar surface $\overline{\partial W-\left(H_{i} \cup H_{j}\right)}$ (with four boundary components) is incompressible in $\overline{W-\left(H_{i} \cup H_{j}\right)}$. Now one is ready to apply [CGLS, Lemma 2.3.2] to see that $W$ must be a punctured lens space.

From now on $\left(L^{0}\left(p, q^{\prime}\right) ; \alpha_{1}, \ldots, \alpha_{k}\right)$ denotes an irreducible non-split completely tubing compressible tangle in a punctured lens space $L^{0}\left(p, q^{\prime}\right)$. We may assume that $p$ be positive. It is well known that two lens spaces $L\left(p, q^{\prime}\right)$ and $L(p, q)$ are homeomorphic if and only if either $q \equiv \pm q^{\prime} \bmod (p)$ or $q q^{\prime} \equiv \pm 1 \bmod (p)$. We may assume that $k \geq 2(k=1$ case is completely determined). By Corollary 2, we may assume, for any fixed $i$ of $1, \ldots, k$, that $L^{0}\left(p, q^{\prime}\right)$ is a union of a solid torus $V=D^{2} \times S^{1}$ and the 2-handle $H_{i}$ whose attaching annulus $A_{i}$ is glued with a regular neighborhood of a $(q, p)$ curve in $\partial V$, with respect to a fixed meridian-longitude basis on $\partial V$. Also within the topological equivalence class of the tangle, we may actually assume that $1 \leq q<p / 2$. This is because that there is a homeomorphism of the solid torus $V$ to itself sending the $(q, p)$ curve in $\partial V$ to the $( \pm q+m p, p)$ curve in $\partial V$ for any given integer $m$. In summary, we may assume that $L^{0}\left(p, q^{\prime}\right)=V \cup_{A_{i}} H_{i}$ and the center circle $c_{i}$ of $A_{i}$ is a $(q, p)$ curve on $\partial V$ with respect to some fixed meridian-longitude basis whose orientations are chosen in such way that they are consistent with the right-hand rule (i.e., if you curve your right hand along the orientation of meridian, then your thumb should point to the orientation of the longitude). Note that a $(q, p)$ curve can be placed on $\partial V$ as indicated in Figure 1 (the curve $c_{i}$ in the figure).

Note that $X=\overline{V-\cup_{j \neq i} H_{j}}$ and $F_{i}=\overline{\partial V-\cup_{j \neq i} H_{j}}$. As $F_{i}$ is completely compressible in $X$, each arc $\alpha_{j}, j \neq i$, is boundary parallel in $V$. For each fixed $j \neq i$, we may arrange $\alpha_{j}$, by isotopy, to be properly embedded in a disk fiber $D$ of $V$. The boundary of $\alpha_{j}$ separates $\partial D$ into two $\operatorname{arcs} \delta$ and $\delta^{\prime}$. Then the number of components of $\delta \cap c_{i}$ and the number of components of $\delta^{\prime} \cap c_{i}$ add to $p$. Let $b_{j}^{i}$ be the minimal of the two numbers. Then $0 \leq b_{j}^{i}<p$. We call $b_{j}^{i}$ the bridge width of the arc $\alpha_{j}$ with respect to the arc $\alpha_{i}$ (it is obviously well defined by its canonical constructional definition).

Proposition 3. Under the setting as established above, we have that for each fixed $i$, the bridge width $b_{j}^{i}=1$ or $q$ for each $j \neq i$. 


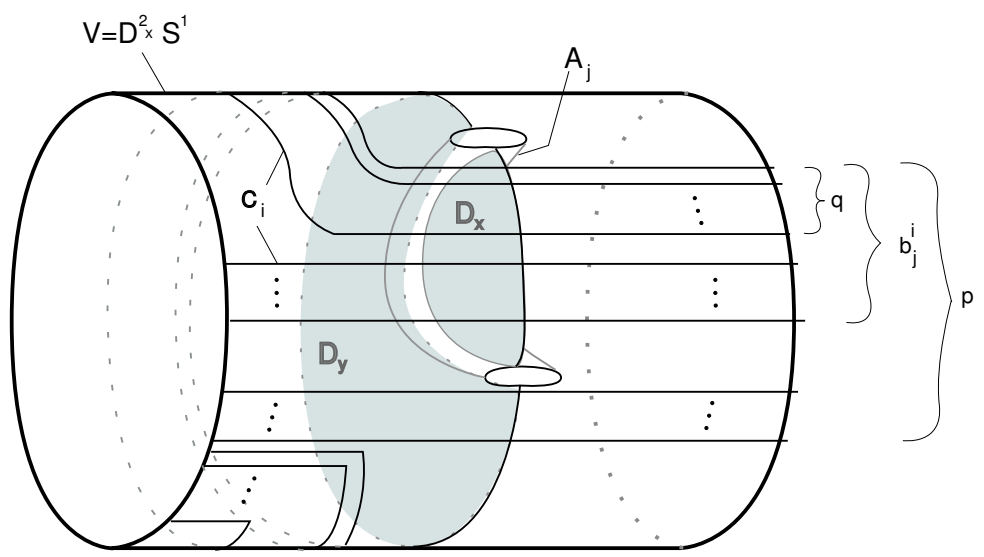

Figure 1. The disks $\left\{D_{x}, D_{y}\right\}$ of the genus two handlebody dual to a free basis $\{x, y\}$.

Proof. Consider the manifold $Y=\overline{V-H_{j}}$ and let $A_{i}^{\prime}=\overline{\partial V-A_{i}}$ and $Q=$ $\overline{A_{i}^{\prime}-H_{j}}$. From the proof of Lemma 1, we knew that $Q$ is incompressible in $Y$. It follows that the bridge width $b_{j}^{i}$ must be positive. Note that $Y$ is a handlebody of genus two as shown by Figure 1. Choose two disks $D_{x}$ and $D_{y}$ as in Figure 1 which cuts $Y$ into a 3-ball. So there is a free basis $\{x, y\}$ for the fundamental group of $Y$ dual to the disk system $\left\{D_{x}, D_{y}\right\}$, i.e., $x$ has a representing loop intersecting $D_{x}$ transversely exactly once and $y$ has a representing loop intersecting $D_{y}$ transversely exactly once. Now attaching the 2-handle $H_{i}$ to $Y$ along $c_{i}$ is a solid torus by Corollary 2. This happens if and only if the word represented by the loop $c_{i}$ in $x$ and $y$ is a free basis element of the free group. Now we are in a setting to apply $[\mathbf{D}$, Theorem 3.2.2] to see that the latter happens if and only if $b_{j}^{i}=1$ or $q$. The proposition is proved.

Proposition 3 gives a complete classification of all irreducible non-split completely tubing compressible tangles with two strands. We also have:

Corollary 4. If $\left(L^{0}(p, 1) ; \alpha_{1}, \ldots, \alpha_{k}\right)$ is an irreducible non-split completely tubing compressible tangle, then any two strands $\alpha_{i}$ and $\alpha_{j}$ from $\left\{\alpha_{1}, \ldots, \alpha_{k}\right\}$ are parallel in $L^{0}(p, 1)$.

Proof. Since $q^{\prime}=1$, we see that for a fixed $i$, the attaching curve of the 2-handle $H_{i}$ to $V$ can be assumed to be the $(1, p)$ curve by the classification of lens spaces (go back to the discussion after Corollary 2). Now according to Proposition 3, each other stand $\alpha_{j}, j \neq i$, has bridge width (with respect 
to $\left.\alpha_{i}\right) b_{j}^{i}=1$. Also $\alpha_{j}$ is boundary parallel in $V$ for each $j \neq i$. It follows obviously that $\alpha_{j}$ is parallel to $\alpha_{i}$ in their embient space $L(p, 1)$.

When there are more than two strands in such tangle, their mutual positions are much more complicated to analyse. We do not even know if $\alpha_{j}$ and $\alpha_{m}$ are parallel in $V-A_{i}$ when $b_{j}^{i}=b_{m}^{i}$. The following conjecture suggests a possible picture of strands in such a tangle.

Conjecture 5. Let $\left(L^{0}\left(p, q^{\prime}\right) ; \alpha_{1}, \ldots, \alpha_{k}\right)$ be an irreducible non-split completely tubing compressible tangle. Then for each fixed $i$, all strands $\alpha_{j}$, $j \neq i$, can be arranged by isotopy in $V-A_{i}$ to form at most two parallel families as shown in Figure 2, one family with bridge width 1 and the other family with bridge width $q$.

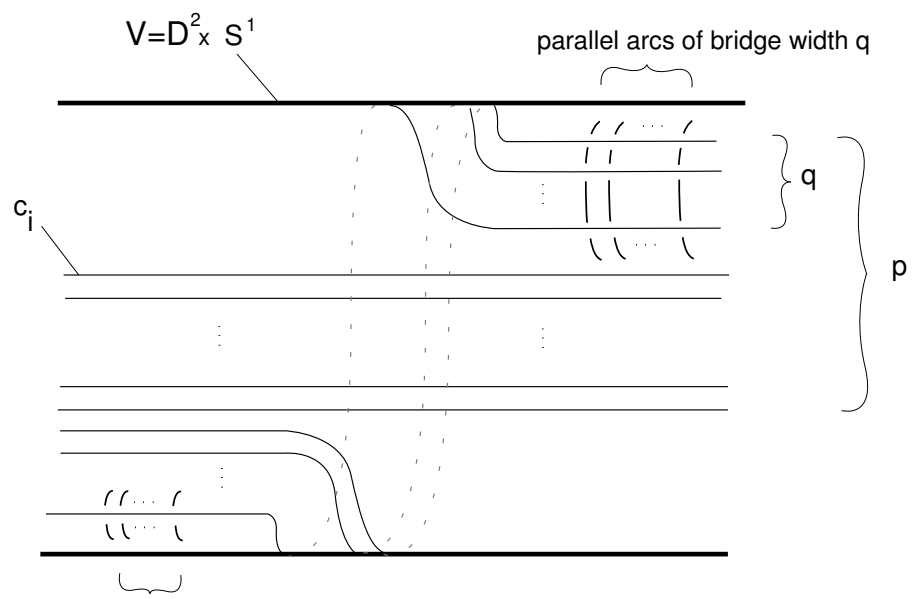

parallel arcs of bridge width 1

Figure 2. A conjectural picture of an irreducible non-split completely tubing compressible tangle.

\section{Knots in solid torus.}

We now give another interesting consequence of Proposition 3, which concerns 1-bridge braid knots in a solid torus. Recall that a non-trivial knot $K$ in a solid torus $V=D^{2} \times S^{1}$ is called a 0 -bridge braid knot (also called torus knot) if it can be isotoped into $\partial V$, and is called a 1-bridge braid knot if it is not a 0-bridge braid knot but can be isotoped as a union of two $\operatorname{arcs} \alpha$ and $\beta$ such that $\alpha$ lies in $\partial V$ intersecting each disk fiber of $V=D^{2} \times S^{1}$ transversely and $\beta$ is properly embedded in a disk fiber of $V$ [Ga2]. In [Ga2], the class of 1-bridge braid knots are classified (up to orientation preserving 
homeomorphisms) by the triple $(\omega, b, t)$, where $\omega$ is the winding number of $K, b$ is the bridge width of $K(1 \leq b \leq \omega-2)$ and $t$ is the twist number of $K$ $(1 \leq t \leq \omega-2)$. Figure 3 explains how a 1-bridge braid knot with winding number 9 , bridge width 2 and twist number 5 is defined (constructed). This example should suffice for seeing how the general triple $(\omega, b, t)$ is defined. We note that the convention of bridge width used here is different from that used [Ga2] by taking the mirror image; that is, the bridge width $b$ used here is the number $\omega-b-1$ in the convention of [Ga2]. Knots in a solid torus which admit a non-trivial surgery yielding a solid torus are classified in $[\mathbf{B}]$. Such a knot must be either a 0-bridge braid knot or a 1-bridge braid knot. However it seems not so easy to see that whether the exterior of a hyperbolic 1-bridge braid knot $K$ in $V$ admits a Dehn filling along $\partial V$ yielding a solid torus. We shall give a class of such knots.

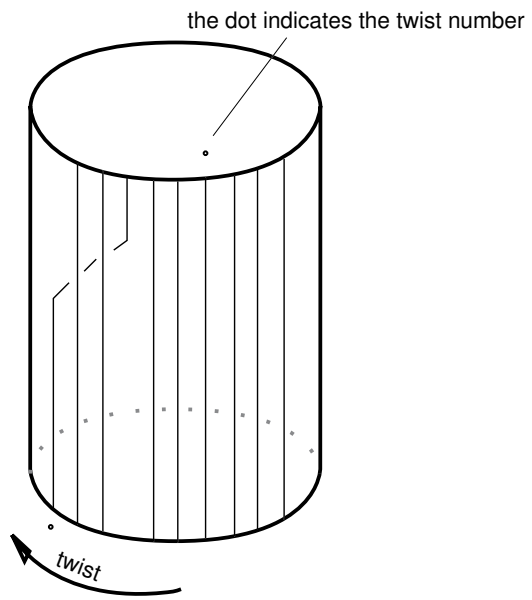

(a)

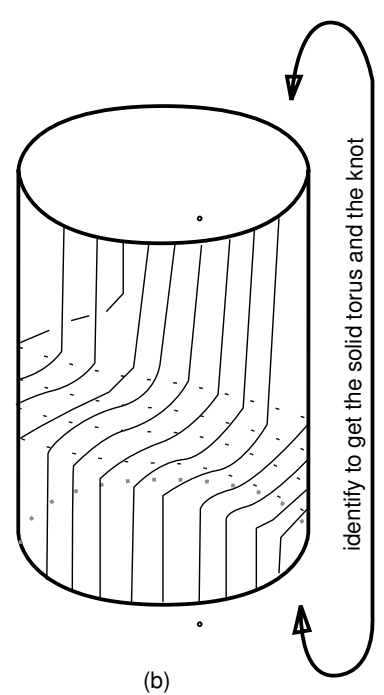

(b)

Figure 3. 1-bridge braid knot with $\omega=9, b=2$ and $t=5$.

Let $V=D^{2} \times S^{1}, K \subset V$ a non-trivial knot (i.e., not contained in a 3-ball in $V$ ), $M$ the exterior of $K$ in $V, T_{0}=\partial V, T_{1}=\partial M-T_{0}$ (we call $T_{0}$ the outer boundary torus of $\left.M\right)$. Fix a basis $\left\{\mu_{0}, \lambda_{0}\right\}$ for $T_{0}$ where $\mu_{0}$ is the meridian slope of $V$. Embed $V$ in $S^{3}$ as a trivial solid torus so that $\lambda_{0}$ bounds a disk in $S^{3}$. Let $\left\{\mu_{1}, \lambda_{1}\right\} \subset T_{1}$ be the standard meridian-longitude basis of $K$ when considered as a knot in $S^{3}$. Slopes on $T_{1}$ and on $T_{0}$ will be parameterized with respect to these bases respectively. For a loop $\gamma$ in a manifold, we use $[\gamma]$ to denote its homology class in the first homology group of the manifold. Again the orientations of $\left[\mu_{0}\right]$ and $\left[\lambda_{0}\right]$ in the outer boundary torus $T_{0}$ follow the right hand rule. We orient $\left[\mu_{1}\right]$ and $\left[\lambda_{1}\right]$ such 
that $\left[\lambda_{1}\right]=\omega\left[\lambda_{0}\right]$ and $\left[\mu_{0}\right]=\omega\left[\mu_{1}\right]$ with $\omega \geq 0$ being the winding number of $K$ in $V$.

Proposition 6. Let $K$ be a 1-bridge braid knot in $V$ defined by the triple $(\omega, b, t)$ and let $M$ be its exterior. If $b<\omega / 2, t=\omega-b-1$ and $(\omega+1, b+1)=$ 1 , then Dehn filling $M$ along $\partial V$ with the slope $\pm\left((b+1)\left[\mu_{0}\right]+(\omega+1)\left[\lambda_{0}\right]\right)$ yields a solid torus and the slope $\pm\left(\omega^{2}(b+1)\left[\mu_{1}\right]+(\omega+1)\left[\lambda_{1}\right]\right)$ on $T_{1}$ becomes the meridian slope of the resulting solid torus.

Proof. A 1-bridge braid knot $K$ satisfying the conditions of the proposition has a representative as shown in Figure 4 (a). Now consider the $(b+1, \omega+1)$ curve $c$ on $\partial V$ and let $\alpha$ be a boundary parallel simple arc in $V$ with bridge width $b+1$ as shown in Figure 4 (b). Let $Y=\overline{V-N(\alpha)}$. By Proposition 3 , attaching a 2-handle to $Y$ along the curve $c$ results a solid torus. On the other hand, one can slide the two end points of $\alpha$ along $\partial V-c$ in the way as indicated in Figure 4 (b). More precisely let $\partial V-c$ have the induced foliation from the disk foliation of $V$ (we may assume that $c$ is transverse to the disk foliation of $V$ ), the sliding of $\alpha$ along $\partial V-c$ is always transverse to the interval leaves of the foliation. When the two end points eventually meet at the same interval leave for the first time, connect them along the interval. The resulting knot is the 1-bridge braid knot $K$ in $V$ (after pushed into the interior of $V$ ) with the triple $(\omega, b, \omega-b-1)$. Now one only needs to observe that the manifold obtained by attaching a 2-handle to $Y$ along $c$ is the same as that obtained by Dehn filling $M$ along $T_{0}$ with the slope represented by $c$. To see the meridian slope of the resulting solid torus, note that $H_{1}(M ; \mathbb{Z})$ is a free rank two abelian group generated by $\left[\mu_{1}\right]$ and $\left[\lambda_{0}\right]$, and $\left[\lambda_{1}\right]=\omega\left[\lambda_{0}\right],\left[\mu_{0}\right]=\omega\left[\mu_{1}\right]$. The Dehn filling along $T_{0}$ gives the homology relation $(b+1)\left[\mu_{0}\right]+(\omega+1)\left[\lambda_{0}\right]=(b+1) \omega\left[\mu_{1}\right]+(\omega+1)\left[\lambda_{0}\right]=0$. Hence a slope $\pm\left(m\left[\mu_{1}\right]+n\left[\lambda_{1}\right]\right)= \pm\left(m\left[\mu_{1}\right]+n \omega\left[\lambda_{0}\right]\right)$ on $T_{1}$ can be the meridian slope of the resulting solid torus only if $m=\omega^{2}(b+1)$ and $n=\omega+1$.

We remark that a 1-bridge braid knot $K$ in a solid torus is not a 0-bridge braid knot and hence the exterior of $K$ is not Seifert fibered. Also if the winding number $\omega$ of a 1-bridge braid knot $K$ is a prime number, then the exterior of $K$ is hyperbolic [GW2, Corollary 7.4]. In fact applying results of [MS] or [E] one can show that a 1-bridge braid knot $K$ in a solid torus $V$ has its exterior containing essential torus if and only if $K$ is an $(1, m)$ cable of a $(q, p)$ torus knot in $V$ (with $|m|>1$ and $|p|>1$ ).

Problem 7. Classify 1-bridge braid knots in a solid torus whose exterior admits Dehn filling along the outer boundary torus $T_{0}$ yielding solid torus.

One may further consider which Dehn filling of $M$ along the outer boundary torus $T_{0}$ gives Seifert fibered space different from solid torus. Our next proposition provides a class of such knots which again include infinitely many hyperbolic knots. 


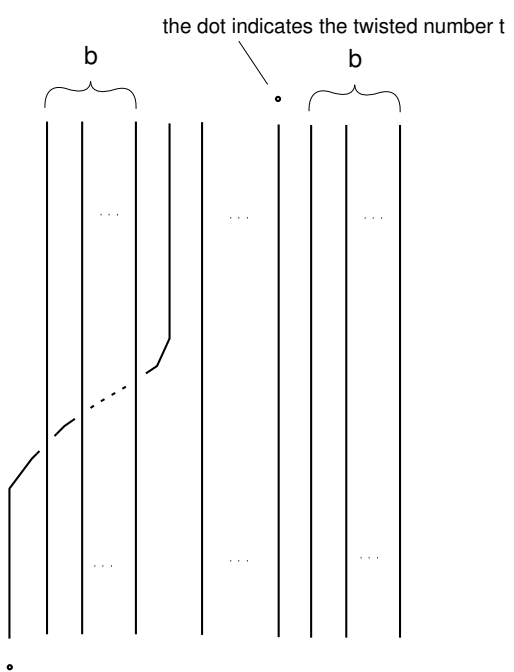

(a)

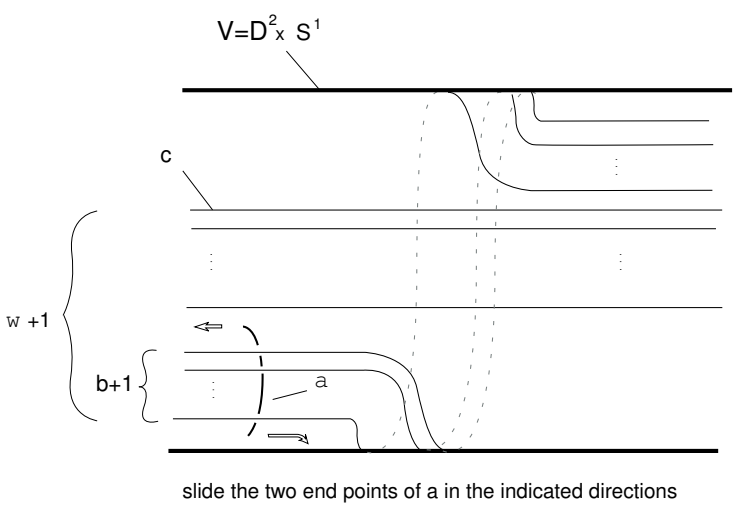

(b)

Figure 4. Slide the arc $\alpha$ in part (b) to obtain the 1-bridge braid knot in part (a).

Proposition 8. Let $K$ be a 1-bridge braid knot in $V$ defined by the triple $(\omega, b, t)$ and let $M$ be its exterior. If for some integer $m>1$ and $n \geq 1$, $b=m n, t=\omega-1-n$ and $(\omega+m, n+1)=1$, then Dehn filling $M$ along $\partial V$ with the slope $\pm\left((n+1)\left[\mu_{0}\right]+(\omega+m)\left[\lambda_{0}\right]\right)$ yields a Seifert fibered space whose base orbifold is a disk with two singular points.

Proof. The argument is similar to that of Proposition 6 but applying another result of $[\mathbf{D}]$. A 1-bridge braid knot $K$ satisfying the conditions of the proposition has a representative as shown in Figure 5 (a). Now consider the $(n+1, \omega+m)$ curve $c$ on $\partial V$ and let $\alpha$ be a boundary parallel simple arc in $V$ with bridge width $n(m+1)$ as shown in Figure $5(\mathrm{~b})$. Let $Y=\overline{V-N(\alpha)}$. By [D, Proposition 3.3.1], attaching a 2-handle to $Y$ along the curve $c$ results a Seifert fibered space whose base orbifold is a disk with two singular points. On the other hand, one can slide the two end points of $\alpha$ along $\partial V-c$ (in directions as indicated in Figure 5 (b)) to get a 1-bridge braid knot $K$ in $V$ such that $K$ is of the given triple $(\omega, m n, \omega-n-1)$. Again the manifold obtained by attaching a 2-handle to $Y$ along $c$ is the same as that obtained by Dehn filling $M$ along the outer boundary torus $T_{0}$ with the slope represented by $c$. 


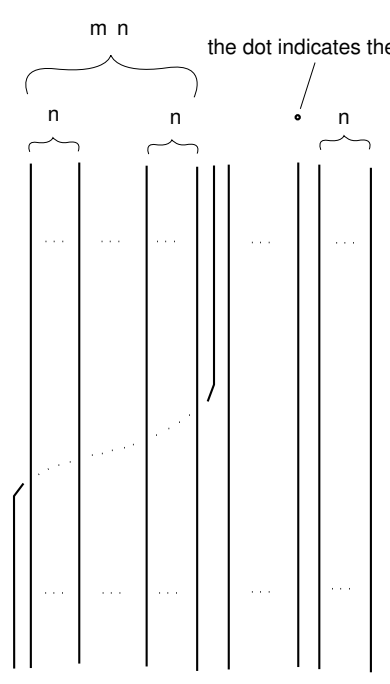

(a)

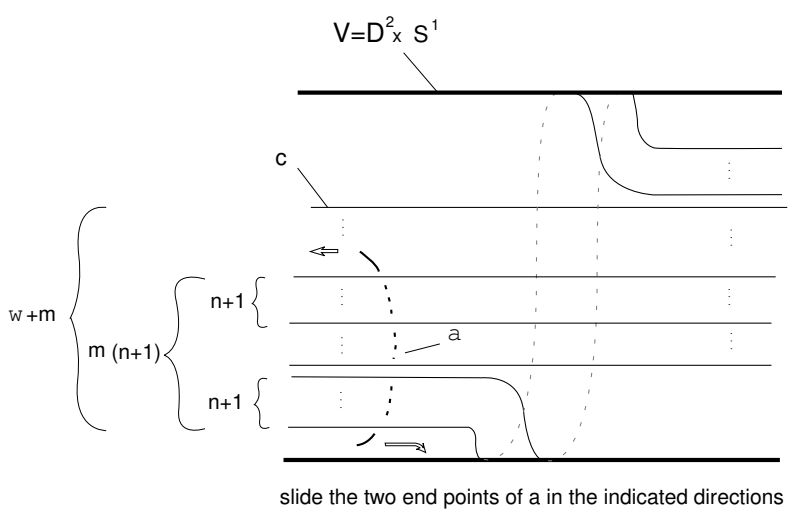

(b)

Figure 5. Slide the arc $\alpha$ in part (b) to obtain the 1-bridge braid knot in part (a).

Here is perhaps a convenient place to make a note on Dehn surgery on a hyperbolic knot in a solid torus resulting a Seifert fibered space.

Proposition 9. Let $M$ be the exterior of a hyperbolic knot $K$ in a solid torus $V$. If some Dehn filling on $M$ along the inner boundary torus $T_{1}$ with slope $\beta$ produces a Seifert fibered space, then $\beta$ is an integer slope.

Proof. Let $U=M\left(T_{1}, \beta\right)$ denote the manifold obtained by Dehn filling $M$ along the inner boundary torus $T_{1}$ with the slope $\beta$. Let $F$ be the base orbifold of a Seifert fibration of $U$. Then $F$ has a single boundary component. We may assume that $F$ is not a disk with at most one cone point since otherwise $U$ is a solid torus in which case $\beta$ is known to be an integer slope.

Suppose that $F$ is a disk with exactly two cone points. Let $\sigma$ be the slope in $T_{0}=\partial U$ represented by a fiber in $T_{0}$ of the Seifert fibration of $U$. One can now choose a slope $\delta$ in $T_{0}$ satisfying the conditions: (1) $\Delta(\delta, \sigma)=1$ (here and later $\Delta$ is used to denote the distance, i.e., the geometric intersection number, of two slopes); (2) The manifold $N=M\left(T_{0}, \delta\right)$, obtained by Dehn filling $M$ along its outer boundary torus $T_{0}$ with the slope $\delta$, is a hyperbolic manifold. Such $\delta$ exists because of the well known hyperbolic surgery theorem of Thurston $[\mathbf{T}]$ which assures that except for finitely many slopes on $T_{0}$, all remaining slopes on $T_{0}$ will produce hyperbolic 3 -manifolds, and because of the fact that there are infinitely many slopes on $T_{0}$ which 
are distance one from a given slope. The manifold $N(\mu)$, obtained by Dehn filling $N$ along $\partial N=T_{1}$ with the meridian slope $\mu$, is the manifold $V(\delta)$, obtained by Dehn filling the solid torus $V$ along $\partial V=T_{0}$ with the slope $\delta$, and thus is a lens space or $S^{3}$ or $S^{2} \times S^{1}$. And the manifold $N(\beta)$, obtained by Dehn filling $N$ along $\partial N=T_{1}$ with the slope $\beta$, is the manifold $U(\delta)$, obtained by Dehn filling $U$ along $\partial U=T_{0}$ with the slope $\delta$, is a Seifert fibered space with a Seifert fiberation whose base orbifold is a 2 -sphere with exactly two cone points since $\Delta(\sigma, \delta)=1$, and thus is a lens space or $S^{3}$ or $S^{2} \times S^{1}$. Hence applying the cyclic surgery theorem of [CGLS] to the hyperbolic manifold $N$, we get $\Delta(\beta, \mu)=1$, i.e., $\beta$ is integer slope.

So we may assume that the base orbifold $F$ is not a disk with at most two cone points. We may also assume that $F$ is not a Mobius band since otherwise $U$ has another Seifert fibration whose base orbifold is a disk with two cone points (of indices 2 and 2) $[\mathbf{J}]$. We still use $\sigma$ to denote the slope in $T_{0}=\partial U$ represented by a fiber in $T_{0}$ of the Seifert fibration of $U$. One can now choose a slope $\delta$ in $T_{0}$ satisfying the conditions: (1) $\Delta(\sigma, \delta)>1 ;(2)$ The manifold $N=M\left(T_{0}, \delta\right)$, is a hyperbolic manifold. Again the manifold $N(\mu)=V(\delta)$ is a lens space or $S^{3}$ or $S^{2} \times S^{1}$. The manifold $N(\beta)=U(\delta)$ is a Seifert fibered space which admits no Seifert fibration with base orbifold being a 2 -sphere with at most three cone points since $\Delta(\sigma, \delta)>1$ implies that the Seifert fibration of $U(\delta)$ coming from an extension of $U$ will have an extra singular fiber which is the core of the filling solid torus. Hence we may apply [BZ2, Theorem 1.5] to the hyperbolic manifold $N$ to conclude that $\Delta(\beta, \mu)=1$.

If a hyperbolic knot in a solid torus admits a non-trivial surgery yielding a solid torus, then the surgery slope is an integer, say $m$ [CGLS], [Ga2]. By Proposition 9, such knot admits at most two other non-trivial surgeries yielding Seifert fibered spaces and the surgery slope(s) must be $m-1$ or/and $m+1$. It is proved in $[\mathbf{B}]$ that there is a unique (up to topological equivalence) hyperbolic knot in a solid torus which admits two non-trivial surgeries yielding solid torus. By Proposition 9, this knot admits no other non-trivial surgery yielding Seifert fibered space.

\section{Tangle sums and the cabling conjecture.}

We now continue our study of tangles and retain notations established earlier. Given two tangles of the same number of strands, $\left(W ; \alpha_{1}, \ldots, \alpha_{k}\right)$ and $\left(W^{\prime} ; \alpha_{1}^{\prime}, \ldots, \alpha_{k}^{\prime}\right)$, and any homeomorphism

$$
h:\left(\partial W ; \cup \partial \alpha_{i}\right) \rightarrow\left(\partial W^{\prime} ; \cup \partial \alpha_{i}^{\prime}\right),
$$

one can construct a manifold pair $(Q, L)$ such that $Q$ is the usual connected sum of $W$ and $W^{\prime}$ and $L$ is a link in $Q$ obtained by identify end points of the strands $\alpha_{1}, \ldots, \alpha_{k}$ with the end points of the strands $\alpha_{1}^{\prime}, \ldots, \alpha_{k}^{\prime}$ 
using the map $h$. We call $(Q, L)$ the $h$-sum of the two tangles. Recall that $X$ (resp. $X^{\prime}$ ) denotes the exterior of the strands $\alpha_{i}$ 's (resp. $\alpha_{i}$ 's) in $W$ (resp. $\left.W^{\prime}\right)$ and $P$ (resp. $\left.P^{\prime}\right)$ is the planar surface $\overline{\partial W-\left(H_{1} \cup \ldots \cup H_{k}\right)}$ (resp. $\left.\overline{\partial W^{\prime}-\left(H_{1}^{\prime} \cup \ldots \cup H_{k}^{\prime}\right)}\right)$. Now the map $h$ induces through restriction a homeomorphism map $\left.h\right|_{P}: P \rightarrow P^{\prime}$ such that the manifold $M=X \cup_{h} X^{\prime}$ is the link exterior of $L$ in $Q$. If both of the tangles are irreducible and nonsplit, then $M$ is an irreducible manifold such that $P$ is an incompressible planar surface properly embedded in $M$, and moreover if in addition both of the tangles are atoroidal and annular, then $M$ is hyperbolic. The question of interest to us is when can $M$ be homeomorphic to a knot exterior in $S^{3}$ and if so must both of $W$ and $W^{\prime}$ be the 3-ball? This is another formulation of the cabling conjecture. The (stronger) cabling conjecture asserts that if $M$ is the exterior of a knot in $S^{3}$, then it has no properly embedded incompressible planar surface with more than two boundary components and with its boundary slope different from the meridian slope of the knot. We remark that if such planar surface, denoted $P$, exists in $M$, then $M\left(r_{0}\right)$ is either a reducible manifold or a lens space, where $r_{0}$ is the boundary slope of $P$ (for a slope $r$ on $\partial M$, we use $M(r)$ to denote the manifold obtained by Dehn filling $M$ along $\partial M$ with the slope $r$ ). This follows from [GL3]. We also note that if $M$ is a knot exterior in $S^{3}$ which is cabled, then $M$ does not contain incompressible properly embedded planar surface with more than two boundary components whose slope is different from the meridian slope.

Proposition 10. Suppose that $\left(W ; \alpha_{1}, \ldots, \alpha_{k}\right)$ and $\left(W^{\prime} ; \alpha_{1}^{\prime}, \ldots, \alpha_{k}^{\prime}\right)$ are irreducible and non-split tangles with $k \geq 2$ strands such that $\left(W ; \alpha_{1}, \ldots, \alpha_{k}\right)$ is $A_{p}$-tubing incompressible for some annulus $A_{p}$ and $\left(W^{\prime} ; \alpha_{1}^{\prime}, \ldots, \alpha_{k}^{\prime}\right)$ is $A_{q^{-}}^{\prime}$ tubing incompressible for some annulus $A_{q}^{\prime}$. Let $(Q, K)$ be any h-sum of the two tangles such that $K$ is a knot. Then the exterior $M$ of $K$ in $Q$ is not homeomorphic to a knot exterior in $S^{3}$ unless both $W$ and $W^{\prime}$ are 3 -balls, in which case the knot $K$ also satisfies the cabling conjecture.

Proof. Let $r_{0}$ be the boundary slope of $P$ in $\partial M$. The idea is to construct an essential branched surface in the interior of $M$ which remains essential in $M(r)$ for all filling slopes $r \neq r_{0}$ (see [GO] for the definition of an essential branched surface and for their topological implications to their ambient manifolds). Hence if one of $W$ and $W^{\prime}$ is not a 3-ball, then the slope $r_{0}$ cannot be the canonical meridian slope of $\partial M$ if $M$ is a knot exterior in $S^{3}$. But then $M$ cannot admit a Dehn filling with a slope different from $r_{0}$ producing the 3 -sphere as $S^{3}$ has no essential branched surface. If both $W$ and $W^{\prime}$ are 3 -balls, then $r_{0}$ is the meridian slope in $\partial M$. But then $M$ cannot admit a Dehn filling producing a reducible 3-manifold or a lens space as a reducible manifold or a lens space has no essential branched surface. Hence in such case, the knot $K$ satisfies the cabling conjecture. 
The construction of the essential branched surface is a generalization of a method given in $[\mathbf{W}]$. The planar surface $(P, \partial P) \subset(M, \partial M)$ has $2 k \geq 4$ boundary components. Up to re-ordering, we may assume that the strands $\alpha_{i}$ and $\alpha_{j}^{\prime}$ occur in the knot $K$ in the order $\alpha_{1}, \alpha_{1}^{\prime}, \alpha_{2}, \alpha_{2}^{\prime}, \ldots, \alpha_{k}, \alpha_{k}^{\prime}$ and we may also assume that $p=1$. Now construct a branched surface $B$ in $M$ as shown in Figure 6. Topologically $B$ is the union of $P$ and the annuli $A_{1}^{\prime}, A_{2}, A_{2}^{\prime}, \ldots, A_{k}, A_{k}^{\prime}$. The boundary circles of $A_{2}, A_{3}, \ldots, A_{k}$ are the branch set of $B$, which we denoted in Figure 6 by $b_{1}, b_{2}, \ldots, b_{2 k-2}$. Make cusps at $b_{1}, \ldots, b_{2 k-2}$ as indicated in Figure 6 . Push $B$ slightly into the interior of $M$ and denote the resulting branched surface still by $B$. Now following $[\mathbf{W}]$ with obvious modifications, one can show that $B$ is essential in $M$ and remains essential in $M(r)$ for all slopes $r \neq r_{0}$.

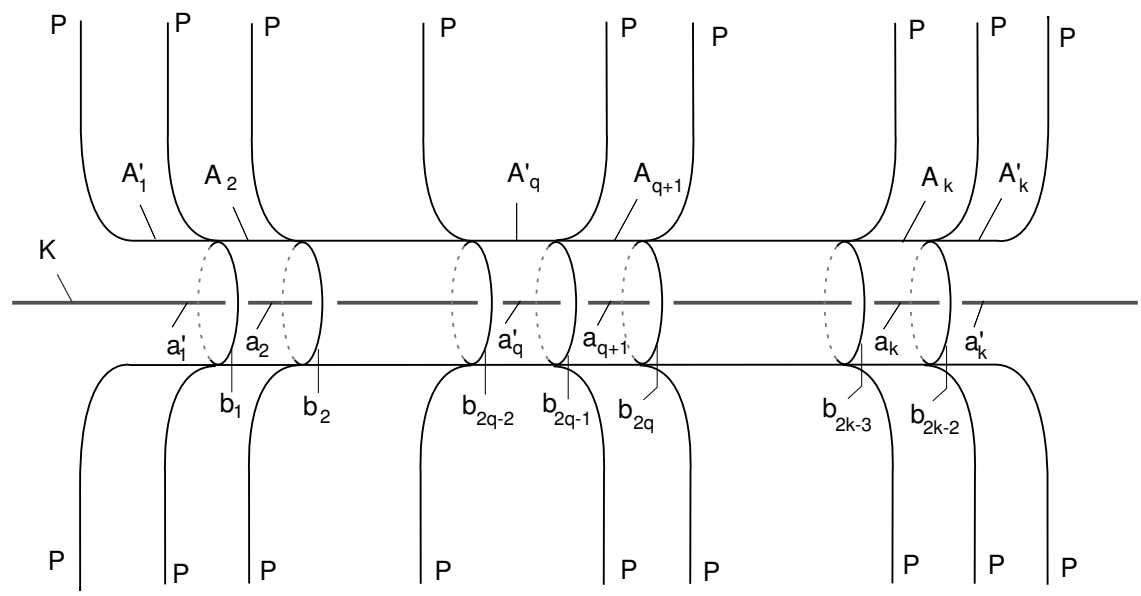

Figure 6. The branched surface $B$ in $M$.

Proposition 11. Suppose that $\left(W ; \alpha_{1}, \alpha_{2}\right)$ and $\left(W^{\prime} ; \alpha_{1}^{\prime}, \alpha_{2}^{\prime}\right)$ are irreducible and non-split tangles. Let $(Q, K)$ be any $h$-sum of the two tangles such that $K$ is a knot. Then the exterior $M$ of $K$ in $Q$ is not homeomorphic to a knot exterior in $S^{3}$ unless both $W$ and $W^{\prime}$ are 3 -balls in which case the knot $K$ also satisfies the cabling conjecture.

Proof. First we suppose that $M$ is homeomorphic to a knot exterior in $S^{3}$ and at least one of $W$ and $W^{\prime}$ is not the 3-ball. We will get a contradiction. In this case, the boundary slope $r_{0}$ of $P$ on $\partial M$ is not the canonical meridian slope. Let $\mu$ be the meridian slope. Then $\Delta\left(\mu, r_{0}\right)$, the geometric intersection number of $\mu$ and $r_{0}$ on $\partial M$, is equal to one by [GL2]. By [GL3], there is a planar surface $(Q, \partial Q) \subset(M, \partial M)$ satisfying the following conditions: (1) Each component of $\partial Q$ is an essential simple loop in $\partial M$ with slope $\mu$; (2) 
$Q$ intersects $P$ transversely such that each component of $\partial Q$ intersects each component of $\partial P$ in exactly one point; (3) No arc component of $Q \cap P$ is boundary parallel in either $Q$ or $P$. The arc components of $Q \cap P$ give rise to two "dual" graphs $\Gamma_{Q}$ and $\Gamma_{P}$ in $Q$ and $P$ respectively. Namely one takes the boundary components of $Q$ (resp. $P$ ) as vertices of $\Gamma_{Q}\left(\right.$ resp. $\left.\Gamma_{P}\right)$ and arc components of $Q \cap P$ as edges of $\Gamma_{Q}$ (resp. $\Gamma_{P}$ ). In our present case, $\Gamma_{P}$ has four vertices coming from the components of $\partial A_{1}$ and $\partial A_{2}$. It follows from [GL3] that the graph $\Gamma_{Q}$ must contain a Scharlemann cycle of order $n>1$ (cf. [GL3] for the definition of Scharlemann cycle). Let $e_{1}, \ldots, e_{n}$ be the edges of the Scharlemann cycle and let $D$ be the disk bounded by the Scharlemann cycle. Then $D$ is properly embedded in $X$ or $X^{\prime}$, say $X$. Further in the surface $P$, the edges $e_{1}, \ldots, e_{n}$ connect two boundary components of $A_{1}$ or $A_{2}$, say $A_{1}$, and divide $P$ into $n>1$ regions.

Now consider the two components of $\partial A_{2}$. If both of them are contained in the same region of $P-\left(e_{1} \cup \ldots \cup e_{n}\right)$, then the complement, denoted by $P^{\prime}$, of this region in $P$ is a disk with two punctures $a_{1}$ and $a_{1}^{\prime}$, containing all the edges $e_{1}, \ldots, e_{n}$. Let $L^{0}$ be a regular neighborhood of $P^{\prime} \cup D \cup H_{1}$ in $W$. Then $L^{0}$ is a punctured lens space of order $n$ since $D$ came from a Scharlemann cycle. Moreover the boundary 2-sphere of $L^{0}$ intersects $\partial M$ exactly twice with the slope $r_{0}$, i.e., $\partial L^{0} \cap M$ is an annulus which we denote by $A$. This annulus $A$ must be essential in $M$, i.e., it cannot be boundary parallel. This follows by considering two sides of $A$ in $M$; one side of $A$ came from the punctured lens space $L^{0}$ and the other side of $A$ contains the incompressible surface $P$ with four boundary components. The existence of such annulus implies that $M$ is cabled. But this gives a contradiction to the early remark immediately prior to Proposition 10 that a cabled knot exterior in $S^{3}$ cannot contain incompressible properly embedded planar surface with more than two boundary components whose slope is different from the meridian slope.

Hence the components of $\partial A_{2}$ are contained in different regions of $P$ $\left(e_{1} \cup \ldots \cup e_{n}\right)$. Let $a_{1}, a_{1}^{\prime}$ be the components of $\partial A_{1}$ and $a_{2}, a_{2}^{\prime}$ the components of $\partial A_{2}$. Note that there are exactly $m$ end points of edges of $\Gamma_{P}$ incident at each of vertices $a_{1}, a_{1}^{\prime}, a_{2}$ and $a_{2}^{\prime}$, where $m$ is the number of boundary components of $Q$. Since no edge in $\Gamma_{P}$ is boundary parallel, edges of $\Gamma_{P}$ which are incident at the vertex $a_{2}$ must connected $a_{2}$ to either $a_{1}$ or $a_{1}^{\prime}$ and thus there are $m$ such edges. Suppose that there are $p$ edges which connect $a_{2}$ to $a_{1}$ and $m-p$ edges which connect $a_{2}$ to $a_{1}^{\prime}$. Similarly we may assume that there are $q$ edges which connect $a_{2}^{\prime}$ to $a_{1}$ and $m-q$ edges which connect $a_{2}^{\prime}$ to $a_{1}^{\prime}$. Also observe that there can be no loops at $a_{1}$ or $a_{1}^{\prime}$. The situation is depicted in Figure 7. Let $n^{\prime}$ be the number of edges of $\Gamma_{P}$ connecting $a_{1}$ and $a_{1}^{\prime}$. Then $n^{\prime} \geq n$. Now counting the end points of edges around $a_{1}$ and $a_{1}^{\prime}$, we get $p+q+n^{\prime}=m$ and $m-p+m-q+n^{\prime}=m$. But these equations imply that $n^{\prime}=0$, a contradiction with $n^{\prime} \geq n>1$. 
So we assume now that both of $W$ and $W^{\prime}$ are 3-balls. Hence $Q=S^{3}$ and the boundary slope of $P$ is the meridian slope $\mu$ of the knot $K$. If the two strands $\alpha_{1}$ and $\alpha_{2}$ in $W$ are parallel, then it is not difficult to see that the tangle $\left(W ; \alpha_{1}, \alpha_{2}\right)$ is toroidal since the tangle is non-split and its ambient space is a 3 -ball. Hence by [W, Lemma 2.1], no Dehn filling on $M$ will produce a reducible manifold or a lens space unless $K$ is a cabled knot. So we may assume that the two stands of the tangle are not parallel. Now by [W, Theorem 2.3] no Dehn filling on $M$ will produce a reducible manifold or a lens space. Hence $K$ satisfies the cabling conjecture.

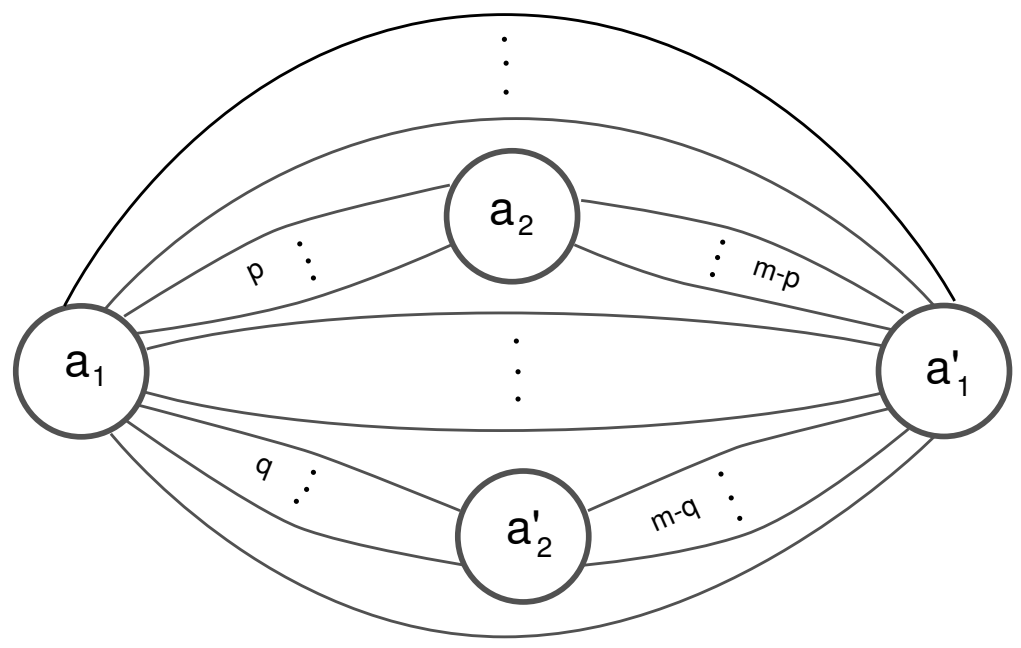

Figure 7. The graph $\Gamma_{P}$.

We note that combining techniques from $[\mathbf{M a}]$ and $[\mathbf{H o}]$, one can show that any knot in $S^{3}$ which admits an irreducible non-split tangle decomposition of at most three strands satisfies the cabling conjecture.

\section{Knots in $S^{3}$ without meridionally-incompressible closed essential surfaces.}

Recall that a closed (embedded) essential (meaning orientable, incompressible and non-boundary parallel) surface $S$ in the exterior $M$ of a knot in $S^{3}$ is said to be meridionally-incompressible if there is no embedded annulus $A$ in $M$ such that $A \cap \partial M$ is one component of $\partial A$ with the meridian slope of the knot and $A \cap S$ is the other component of $\partial A$. Note that a knot in this class is either a composite knot, or a torus knot or a hyperbolic knot. In this section we make some notes concerning Dehn surgery on knots with no meridionally-incompressible closed essential surfaces. Our first observation concerns the cabling conjecture on this class of knots. 
Proposition 12. Let $M$ be the exterior of a non-trivial knot in $S^{3}$ without meridionally-incompressible closed essential surfaces. Suppose that $M$ contains a properly embedded essential planar surface $P$ whose boundary slope $r_{0}$ is not the meridian slope. Then $M\left(r_{0}\right)$ is a connected sum of two lens spaces.

Proof. According to [GL3], $M\left(r_{0}\right)$ contains a lens space as summand and $r_{0}$ is an integer slope but not the longitude slope. So $M\left(r_{0}\right)$ is either a lens space or a reducible manifold. As $r_{0}$ is a boundary slope, [CGLS, Theorem 2.0.3] implies that either $M\left(r_{0}\right)$ is a connected sum of two lens spaces, in which case we are done, or $M$ contains a closed essential surface $S$ such that $S$ remains incompressible in $M(r)$ for all slopes satisfying $\Delta\left(r, r_{0}\right)>1$. We claim that $S$ must be compressible in $M\left(r_{0}\right)$. This is clearly true if $M\left(r_{0}\right)$ is a lens space. In case that $M\left(r_{0}\right)$ is reducible and $S$ is incompressible in $M\left(r_{0}\right)$, one can apply $[\mathbf{S c h}]$ and show that $M$ is cabled. Since $M$ contains essential closed surface, $M$ cannot be a torus knot exterior. Therefore the cabled manifold $M$ must contain an essential torus and thus must be a composite knot exterior. But it is known that a composite knot does not admit surgery producing a lens space or reducible manifold $[\mathbf{G}]$. This contradiction completes the proof of the claim that $S$ is compressible in $M\left(r_{0}\right)$.

By our assumption, $S$ is meridianly compressible. This, together with the condition that $S$ is compressible in $M\left(r_{0}\right)$, implies, by [CGLS, Theorem 2.4.3], that $S$ is compressible in $M(r)$ for all integer slopes $r$. But most of these integer slopes $r$ satisfy $\Delta\left(r, r_{0}\right)>1$. This gives a contradiction to an early conclusion.

Hence, any reducible manifold resulting from Dehn filling a non-trivial knot exterior without meridionally-incompressible closed essential surfaces must be a connected sum of two lens spaces. We remark that by [GS], if a knot exterior in $S^{3}$ admits a filling producing a connected sum of two lens spaces, then the Alexander polynomial of the knot is divisible by the Alexander polynomial of a non-trivial torus knot.

Our next result concerns surgery producing Seifert fibered 3-manifolds. For convenience, in this paper we call a Seifert fibered 3-manifold large if its base orbifold is not a 2 -sphere with at most 4 cone points or a projective plane with at most 2 cone points. It is conjectured that no knot in $S^{3}$ admits a surgery yielding a large Seifert fibered 3-manifold. We note, in contrast, that many of Seifert fibered spaces whose base orbifolds are 2-sphere with at most 4 cone points can be obtained by Dehn surgery on knots in $S^{3}$ [D], $[\mathrm{KT}],[\mathrm{MM}]$.

Proposition 13. Let $M$ be the exterior of a knot without meridionallyincompressible closed essential surfaces. Then no Dehn filling of $M$ produces a large Seifert fibered space. 
Proof. Suppose otherwise that for some slope $r_{0}, M\left(r_{0}\right)$ is a large Seifert fibered space. Then the $P S L(2, \mathbb{C})$-character variety $\bar{X}\left(M\left(r_{0}\right)\right)$ of $M\left(r_{0}\right)$ (see $[$ BZ2] for the definition of the $P S L(2, \mathbb{C})$-character varieties of manifolds and for their basic properties) is at least two dimensional (such estimation of dimension can be found in [BZ2, Section 8]). Note that $\bar{X}\left(M\left(r_{0}\right)\right)$ is naturally contained in $\bar{X}(M)$, the $P S L(2, \mathbb{C})$-character variety of $M$. Recall that each element $\chi_{\bar{\rho}}$ in $\bar{X}(M)$ is the character of a $P S L(2, \mathbb{C})$ representation $\bar{\rho}$ of $\pi_{1}(M)$ and that each element $\gamma \subset \pi_{1}(M)$ defines a regular function $f_{\gamma}$ on $\bar{X}(M)$, whose value at a character $\chi_{\bar{\rho}}$ of $X(M)$ is equal to $\left[\operatorname{trace}\left(\Phi^{-1}(\bar{\rho}(\gamma))\right)\right]^{2}-4$ where $\Phi$ is the canonical quotient map from $S L(2, \mathbb{C})$ to $P S L(2, \mathbb{C})$ (see $[\mathbf{B Z 2}$, p. 759$]$ for details). The condition that $\bar{X}\left(M\left(r_{0}\right)\right) \subset \bar{X}(M)$ is at least two dimensional implies that there is an algebraic curve $X_{0}$ in $\bar{X}\left(M\left(r_{0}\right)\right) \subset \bar{X}(M)$ on which all the functions $f_{r}$, $r \in \pi_{1}(\partial M) \subset \pi_{1}(M)$, are constant functions. This implies that there is an essential closed surface $S$ in $M$ associated to an ideal point of $\tilde{X}_{0}$, where $\tilde{X}_{0}$ is the smooth projective completion of $X_{0}$ (see [BZ2, Proposition 4.7]).

We claim that the surface $S$ must be compressible in the Seifert fibered space $M\left(r_{0}\right)$. For otherwise $S$ is either isotopic to a vertical torus (here vertical means consisting of fibers of the Seifert fibration of $M\left(r_{0}\right)$ ) or to a horizontal surface (meaning transverse to all fibers of the Seifert fibration of $\left.M\left(r_{0}\right)\right)$. Such isotopy of $S$ can be arranged in $M \subset M\left(r_{0}\right)$. If $S$ is a horizontal surface, then it is either a non-separating surface or splits $M\left(r_{0}\right)$ into two twisted $I$-bundles over a closed non-orientable surface. But no knot exterior in $S^{3}$ can contain either a non-separating closed orientable surface, or a closed non-orientable surface. Hence, $S$ must be a vertical torus. It follows that $M$ is the exterior of a satellite knot in $S^{3}$ and, thus, is the exterior of a composite knot in $S^{3}$. But Seifert surgery on composite knots have been classified in $[\mathbf{K T}]$. Namely, if a surgery on a composite knot produces a Seifert fibered space, then the knot is a connected sum of two torus knots and the base orbifold of the Seifert fibered space is a 2-sphere with 4 cone points. But the existence of such a Seifert fiberation contradicts the definition of large. This contradiction completes the proof of the claim that $S$ is compressible in $M\left(r_{0}\right)$.

Recall that $S$ was associated to an ideal point of the curve $\tilde{X}_{0}$ and every function $f_{r}, r \in \pi_{1}(\partial M)$, was bounded (in fact constant) near the ideal point. Further, by construction, for each $\chi_{\bar{\rho}} \in X_{0}, \bar{\rho}\left(r_{0}\right)=I$, since $X_{0} \subset \bar{X}\left(M\left(r_{0}\right)\right) \subset \bar{X}(M)$. Hence, we can apply [BZ2, Proposition 4.10] to conclude that the surface $S$ remains incompressible in $M(r)$, if $\Delta\left(r, r_{0}\right)>1$. In particular, $r_{0}$ is an integer slope since $S$ must be compressible in $M(\mu)$, where $\mu$ is the meridian slope in $\partial M$.

We can now get a contradiction exactly as we did in the last paragraph of the proof of Proposition 12. 


\section{Seifert filling versus other types of exceptional fillings.}

Let $M$ be a compact orientable simple 3-manifold with at least two boundary components one of which is a torus, denoted by $T$. Here the term simple means that $M$ contains no essential 2-sphere; essential annulus; essential torus; or $\partial$-reducing disk. It is shown in [W2] that if one Dehn filling on $M$ along $T$ with slope $\alpha$ produces a reducible manifold and another filling $M$ along $T$ with slope $\beta$ produces a manifold containing essential annulus, then $\Delta(\alpha, \beta) \leq 2$; it is shown in [GW] that if one Dehn filling on $M$ along $T$ with slope $\alpha$ produces a $\partial$-reducible manifold and another filling $M$ along $T$ with slope $\beta$ produces a manifold containing essential annulus, then $\Delta(\alpha, \beta) \leq 2$; it is shown in [W3] that two Dehn fillings on $M$ along $T$ with slopes $\alpha$ and $\beta$ produce $\partial$-reducible manifolds, then $\Delta(\alpha, \beta) \leq 1$; and it is shown in $[\mathbf{S c h}]$ that if one Dehn filling on $M$ along $T$ with slope $\alpha$ produces a reducible manifold, then there is no filling along $T$ that can produce a $\partial$ reducible manifold. We wish to consider Seifert Dehn filling on $M$ along $T$, i.e., fillings resulting in a Seifert fibered space. Note that if some filling on $M$ along $T$ produces a Seifert fibered space, then $\partial M$ must consist of tori and the resulting Seifert fibered space is irreducible (since the manifold has boundary) and contains an essential annulus unless it is a solid torus. Hence it follows from the results cited above that if one Dehn filling on $M$ along $T$ with slope $\alpha$ produces a reducible or $\partial$-reducible manifold, and another filling $M$ along $T$ with slope $\beta$ produces a Seifert fibered space, then $\Delta(\alpha, \beta) \leq 2$. The purpose of this section is to sharpen the bound from 2 to 1. As we mentioned in the introduction section, the bound 1 is best possible.

Proposition 14. Let $M$ be a compact orientable simple 3-manifold whose boundary has at least two components one of which is a torus, denoted by $T$. If one Dehn filling on $M$ along $T$ with slope $\alpha$ yields a reducible manifold and another Dehn filling on $M$ along $T$ with slope $\beta$ yields a Seifert fibered space, then $\Delta(\alpha, \beta) \leq 1$.

Proof. As we already noted, $\partial M$ must consist of tori. (Hence the condition that $M$ is simple is equivalent to the condition that $M$ admits a complete hyperbolic structure of finite volume, according to Thurston $[\mathbf{T}])$. By $[\mathbf{W} 2$, Theorem 4.6] we may assume that $H_{2}(M, \partial M-T)=0$. It follows that $\partial M$ consists of exactly two tori, one is $T$ and the other we denote by $T_{0}$. It also follows that $M$ does not contain any non-separating closed orientable surface. We denote by $M(T, \delta)$ the manifold obtained by Dehn filling $M$ along $T$ with slope $\delta$ in $T$; by $M\left(T_{0}, \delta\right)$ the manifold obtained by Dehn filling $M$ along $T_{0}$ with slope $\delta$ in $T_{0}$; and by $M\left((T, \delta),\left(T_{0}, \eta\right)\right)$ the manifold obtained by Dehn filling $M$ along $T$ with slope $\delta$ in $T$ and along $T_{0}$ with slope $\eta$ in $T_{0}$. If $W$ is a 3 -manifold whose boundary is a single torus, we always use $W(\delta)$ to denote the manifold obtained by Dehn filling $W$ along $\partial W$ with slope $\delta$ in $\partial W$. 
Let $P$ be a non-trivial connect summand of the reducible manifold $M(T, \alpha)$ such that $P$ does not contain $T_{0}$. We have $M(T, \alpha)=P \# Y$, where $Y$ is a 3 -manifold whose boundary is $T_{0}$. There are three cases to consider.

Case 1. $P$ is not a lens space.

Then we can choose a slope $\delta$ in $T_{0}$ such that it satisfies the following conditions: (1) $N=M\left(T_{0}, \delta\right)$ is a hyperbolic 3-manifold; (2) The first Betti number of $N=M\left(T_{0}, \delta\right)$ is one; $(3) M\left(\left(T_{0}, \delta\right),(T, \alpha)\right)$ is a reducible manifold different from $S^{2} \times S^{1}$; (4) There is no essential surface in $M$ disjoint from $T$ but intersecting $T_{0}$ with boundary slope $\delta$ in $T_{0}$. Condition (1) is guaranteed by Thurston's hyperbolic surgery theorem. Condition (2) can be easily satisfied because the first Betti number of $M$ is two (since $H_{2}(M, \partial M-T)=0$ ) and thus all slopes, except for one, in $T_{0}$ will produce manifolds with first Betti number equal to one. As $M\left(\left(T_{0}, \delta\right),(T, \alpha)\right)=P \# Y(\delta)$ and $Y(\delta)$ is not a 3-ball for infinitely many slopes $\delta$ in $T_{0}=\partial Y$, condition (3) follows. Condition (4) is realized by applying the main result of [Ha] which implies that for our manifold $M$, there are only finitely many slopes in $T_{0}$ which can be boundary slopes of essential surface in $M$ disjoint from $T$.

Since $N=M\left(T_{0}, \delta\right)$ is irreducible but $N(\alpha)=M\left(\left(T_{0}, \delta\right),(T, \alpha)\right)$ is reducible by condition (3), $\alpha$ is a boundary slope of $N$. Since the first Betti number of $N$ is one by condition $(2)$ and $N(\alpha)$ is reducible but is not $S^{2} \times S^{1}$ or a connected sum of two lens spaces (since $P$ is not a lens space by our assumption), we may apply [CGLS, Theorem 2.0.3] to see that $N$ must contain an essential closed surface $S$ which remains incompressible in $N(\eta)=M\left(\left(T_{0}, \delta\right)(T, \eta)\right)$ for all slopes $\eta$ in $T$ satisfying $\Delta(\alpha, \eta)>1$. By condition (4), we may assume that the closed essential surface $S$ is disjoint from $T_{0}$, i.e., we have $S \subset M$. Since $M$ is hyperbolic $S$ is of genus at least two.

Claim. $S$ must be compressible in $M(T, \beta)$.

Suppose otherwise. Then $S$ is isotopic to either a horizontal or vertical surface in the Seifert fibered space $M(T, \beta)$. But $S$ cannot be isotopic to a horizontal surface since it is disjoint from $\partial M(T, \beta)=T_{0}$. Neither can $S$ be isotopic to a vertical surface since $S$ is closed and is not a torus. This proves the claim.

Since $S$ is compressible in $M(T, \beta) \subset N(\beta)$, we have $\Delta(\alpha, \beta) \leq 1$. Hence Proposition 14 holds in Case 1.

Case 2. $Y$ is not a solid torus.

Then we can choose a slope $\delta$ in $T_{0}$ such that it satisfies the following conditions: (1) $N=M\left(T_{0}, \delta\right)$ is a hyperbolic 3-manifold; (2) The first Betti number of $N=M\left(T_{0}, \delta\right)$ is one; (3) The fundamental group of $Y(\delta)$ is not cyclic; (4) There is no essential surface in $M$ disjoint from $T$ but with boundary slope $\delta$ in $T_{0}$. Conditions (1) (2) (4) can be justified exactly as in 
Case 1. Condition (3) can also be arranged to hold due to the fact that $Y$ is not a solid torus. So $N(\alpha)=P \# Y(\delta)$ is a reducible manifold but is not $S^{2} \times S^{1}$ or a connected sum of two lens spaces. Now the rest of the proof in this case goes similarly as in Case 1 .

Case 3. $P$ is a lens space and $Y$ is a solid torus.

The idea of proof is similar to that of Proposition 9, but more cases are involved. Let $U=M(T, \beta)$, which is Seifert fibered. Let $F$ be the base orbifold of a Seifert fibration of $U$. We may assume that $F$ is not a disk with at most one cone point since otherwise $U$ is a solid torus in which case the manifold $M$ must be cabled by [Sch], giving a contradiction with the assumption that $M$ is simple. Suppose that $F$ is a disk with exactly two cone points. Let $\sigma$ be the slope in $T_{0}=\partial U$ represented by a fiber in $T_{0}$ of the Seifert fibration of $U$. One can now choose a slope $\delta$ in $T_{0}$ satisfying the conditions: (1) $\Delta(\sigma, \delta)=1 ;(2)$ The manifold $N=M\left(T_{0}, \delta\right)$ is a hyperbolic manifold. It follows from condition (1) that the manifold $N(\beta)$ has cyclic fundamental group since the manifold is a Seifert fibered space which has a Seifert fibration whose base orbifold is a 2-sphere with exactly two cone points. The manifold $N(\alpha)=P \# Y(\alpha)$ is either a lens space (when $Y(\alpha)$ is $S^{3}$ ) or a reducible manifold (when $Y(\alpha)$ is not $S^{3}$ ). If $N(\alpha)$ is a lens space, then we can apply the cyclic surgery theorem of [CGLS] to get $\Delta(\alpha, \beta) \leq 1$; and if $N(\alpha)$ is reducible, then we can apply [BZ2, Theorem 1.2] to get $\Delta(\alpha, \beta) \leq 1$. So we may assume that the base orbifold $F$ is not a disk with at most two cone points. We may also assume that $F$ is not a Mobius band since otherwise $U$ has another Seifert fibration whose base orbifold is a disk with two cone points.

If $F$ is not a 2-disk with three cone points, then we can choose a slope $\delta$ in $T_{0}$ satisfying the conditions: (1) $\Delta(\delta, \mu)=1$ where $\mu \subset T_{0}=\partial Y$ is the meridian slope of the solid torus $Y$; $(2)$ The manifold $N=M\left(T_{0}, \delta\right)$ is a hyperbolic manifold, (3) $\delta$ is not the slope $\sigma$ (the fiber). Condition (1) implies that $N(\alpha)=P \# Y(\alpha)=P$ is a lens space. Also $N(\beta)$ is a Seifert fibered space which admits no Seifert fibration with base orbifold being a 2sphere with at most three cone points. Hence we may apply [BZ2, Theorem 1.5 (1)] to the hyperbolic manifold $N$ to conclude that $\Delta(\alpha, \beta) \leq 1$.

So we may assume that $F$ is a 2-disk with exactly three cone points. If $\sigma$ is not the meridian slope $\mu$ of $Y$ in $\partial Y=T_{0}$, then we can choose a slope $\delta$ in $T_{0}$ satisfying the conditions: (1) $\Delta(\mu, \delta)=1$; (2) The manifold $N=M\left(T_{0}, \delta\right)$ is a hyperbolic manifold; $(3) \Delta(\sigma, \delta)>1$. Condition (1) implies that $N(\alpha)=P$ is a lens space. Condition (3) implies that $N(\beta)$ is a Seifert fibered space which admits no Seifert fibration with base orbifold being a 2-sphere with at most three cone points. Hence we may apply [BZ2, Theorem 1.5] to the hyperbolic manifold $N$ to conclude that $\Delta(\alpha, \beta) \leq 1$. 
Finally suppose that $\sigma$ is the meridian slope of $Y$. Then $M\left((T, \alpha),\left(T_{0}, \sigma\right)\right)$ $=P \# Y(\sigma)=P \# S^{2} \times S^{1}$ and $M\left((T, \beta),\left(T_{0}, \sigma\right)\right)=U(\sigma)$ is a connected sum of three lens spaces since the base orbifold $F$ of the Seifert fibered space $U$ is a 2-disk with exactly three cone points. Let $N=M\left(T_{0}, \sigma\right)$. If $N$ is irreducible, then we may apply the main result of $[\mathbf{G L}]$ to see that $\Delta(\alpha, \beta) \leq 1$ since $N$ admits two fillings each yielding a reducible manifold. So we assume that $N$ is reducible. We may write $N$ as $N=N_{1} \# P_{1}$ with $N_{1}$ being irreducible and contains $T=\partial N=\partial N_{1}$. Since $N(\alpha)$ is a connected sum of a lens space and $S^{2} \times S^{1}, P_{1}$ is either a lens space or $S^{2} \times S^{1}$ or a connected sum of a lens space and $S^{2} \times S^{1}$. But $P_{1}$ cannot contain $S^{2} \times S^{1}$ since otherwise $N(\beta)=M\left((T, \beta),\left(T_{0}, \sigma\right)\right)$ would also contain $S^{2} \times S^{1}$, contradicting the fact that $N(\beta)$ was a connected sum of three lens spaces. So $P_{1}$ must be a lens space. Then $N_{1}(\alpha)=S^{2} \times S^{1}$ and $N_{1}(\beta)$ is a connected sum of two lens spaces. So we can apply $[\mathbf{G L}]$ to $N_{1}$ to get $\Delta(\alpha, \beta)=1$. This completes the proof of Case 3 and thus completes the proof of the proposition.

Corollary 15. Let $M$ be a compact orientable simple 3-manifold whose boundary has at least two components one of which is a torus, denoted by $T$. If one Dehn filling on $M$ along $T$ with slope $\alpha$ yields a $\partial$-reducible manifold and another Dehn filling on $M$ along $T$ with slope $\beta$ yields a Seifert fibered space, then $\Delta(\alpha, \beta) \leq 1$.

Proof. Again $\partial M$ must consist of tori. If $M(T, \alpha)$ is reducible, then we may apply Proposition 14 to get $\Delta(\alpha, \beta) \leq 1$. So we may assume that $M(T, \alpha)$ is irreducible. As $M(T, \alpha)$ is $\partial$-reducible and its boundary consists of tori, it must be a solid torus. Hence we may apply Proposition 9 to see that $\Delta(\alpha, \beta) \leq 1$.

Proposition 16. Let $M$ be a compact orientable simple 3-manifold whose boundary has at least two components one of which is a torus, denoted by $T$. If one Dehn filling on $M$ along $T$ with slope $\alpha$ yields a manifold which contains an essential torus or essential annulus, and another Dehn filling on $M$ along $T$ with slope $\beta$ yields a Seifert fibered space, then $\Delta(\alpha, \beta) \leq 3$.

Proof. Since $M(\beta)$ is Seifert fibered, it is either a solid torus or contains an essential annulus. If $M(\beta)$ is a solid torus, then $\Delta(\alpha, \beta) \leq 2$ by [GW] and [GL5]. So we may assume that $M(\beta)$ contains an essential annulus. By [GW2] and [GW3], $\Delta(\alpha, \beta) \leq 3$ unless $M$ is one of the three manifolds $M_{1}, M_{2}$ and $M_{3}$ given in [GW2, Section 7] and $\Delta(\alpha, \beta)=4$ or 5. More precisely, if $M=M_{1}$ (which is the exterior of the Whitehead link in $S^{3}$ ) and $\Delta(\alpha, \beta)>3$, then $\Delta(\alpha, \beta)=4$ by [GW2, Theorem 1.1] and $M_{1}(\beta)$ is the double branched cover of one the two the tangles given in Figure 7.2 (d) and (e) of [GW2] (see [GW2, Lemma 7.1]). But one can easily check that the double branched cover of each of these two tangles is not Seifert fibered. So $M \neq M_{1}$. Similarly, if $M=M_{2}$ (which is the exterior in $S^{3}$ of 
the link given in [GW2, Figure $7.1(\mathrm{~b})]$ and $\Delta(\alpha, \beta)>3$, then $\Delta(\alpha, \beta)=4$ by [GW2, Theorem 1.1] and $M_{2}(\beta)$ is the double branched cover of one the two tangles given in Figure $7.4(\mathrm{~d})$ and (e) of [GW2] (see [GW2, Lemma 7.5]). But again one can verify that the double branched cover of each of these two tangles is not Seifert fibered. So $M \neq M_{2}$. Finally, if $M=M_{3}$ (which is the exterior in $S^{3}$ of the link given in [GW2, Figure 7.1 (c)]) and $\Delta(\alpha, \beta)>3$, then $\Delta(\alpha, \beta)=5$ by [GW2, Theorem 1.1] and $M_{3}(\beta)$ is the double branched cover of the tangle given in Figure $7.5(\mathrm{~d})$ or $(\mathrm{e})$ of [GW2] (see [GW2, Lemma 7.5]). But again one can verify that the double branched cover of each of these two tangles is not Seifert fibered.

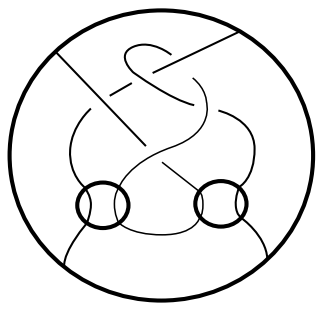

(a)

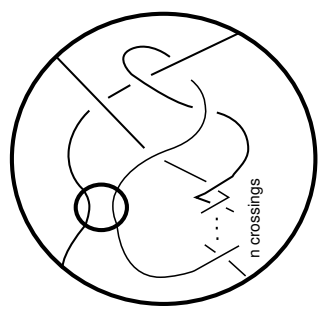

(b)

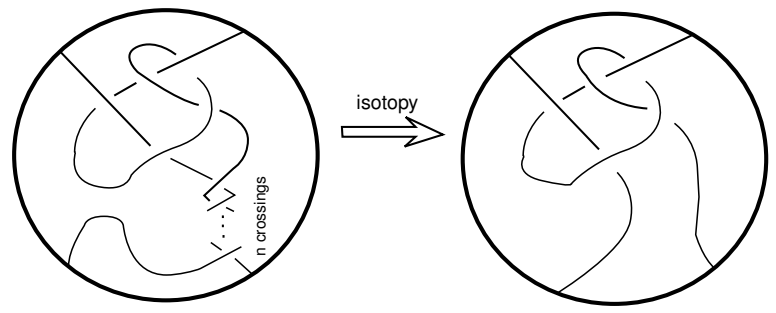

(c)

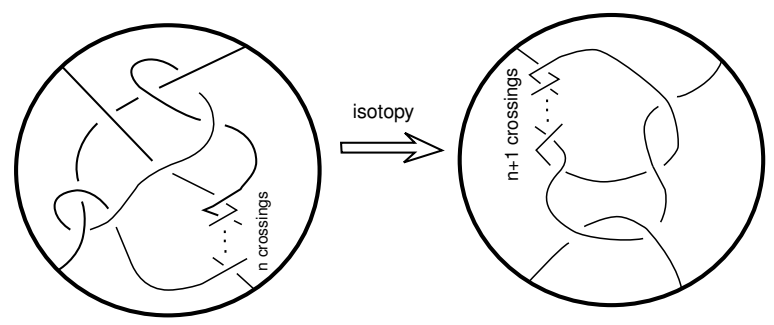

(d)

Figure 8. Distance three between a Seifert slope and an annular and toroidal slope.

Example 17. We give here a family of infinitely many hyperbolic manifolds $N_{n}$ with $\partial N_{n}$ consists of two tori such that one of the tori contains two slopes, distance three apart, one producing a Seifert fibered manifold and the other producing a manifold containing an essential torus and an essential annulus. These examples are constructed based on [GW2, Lemma 7.2]. Here are the details. The manifold $M_{3}$ mentioned in the proof of Proposition 16 is the double branched cover of a twice punctured 3-ball $X$ whose branched set is a set of proper arcs shown in Figure 8 (a) (which is from [GW2, Figure 7.6 (c)]). $M_{3}$ is hyperbolic with $\partial M_{3}$ consists of three tori, which we denote 
by $T_{1}, T_{2}, T_{3}$. In Figure 8 (a), we have chosen framings for the two inside 2 -spheres $S_{1}$ (the one on the left) and $S_{2}$ (the one on the right). We may assume that $T_{1}$ covers $S_{1}$ and $T_{2}$ covers $S_{2}$. Framings on $T_{1}$ and $T_{2}$ are lifts of those on $S_{1}$ and $S_{2}$ respectively. Now we let $N_{n}$ be the manifold obtained by Dehn filling $M_{3}$ along $T_{2}$ with slope $1 / n$. Then it follows from Thurston's hyperbolic surgery theorem that the manifolds $N_{n}$ 's are hyperbolic and are mutually different for infinitely many choices of $n$ (because that in the core of the filling solid torus is a geodesic whose length becomes arbitrarily small as $n$ becomes large). Each $N_{n}$ has its boundary consisting of two tori and is the double branched cover of a once-punctured 3-ball $Y_{n}$ obtained by filling $X$ along $S_{2}$ with a $1 / n$-tangle, as shown in Figure 8 (b). Now Dehn filling $N_{n}$ along $T_{2}$ with the 0 -slope will produces a Seifert fibered space (which is homeomorphic to the trefoil knot exterior in $S^{3}$ ) since the resulting manifold is the double branched cover of the tangle shown in Figure 8 (c), obtained by filling $S_{1}$ with a 0 -tangle. On the other hand, Dehn filling $N_{n}$ along $T_{2}$ with the $-3 / 2$-slope will produces a manifold which contains an essential torus and an essential annulus since the resulting manifold is the double branched cover of the tangle shown in Figure 8 (d), obtained by filling $S_{1}$ with the $-3 / 2$-tangle.

There remains unsettled the case concerning the optimal bound on the distance between two Seifert Dehn filling slopes on a torus boundary component $T$ of a compact orientable simple manifold $M$ with at least two boundary components. Namely what is the minimal upper bound on $\Delta(\alpha, \beta)$ if both $M(T, \beta)$ and $M(T, \alpha)$ are Seifert fibered manifolds? The best known bound is 3 obtained recently in [GW3].

Conjecture 18. The optimal bound is 2 .

The distance two can be realized on the Whitehead link exterior $M$; both the 1-slope and the 3 -slope on any component of $\partial M$ (with respect to the standard framings) produce Seifert fibered spaces.

\section{References}

[A] C. Adams, Toroidally alternating knots and links, Topology, 33 (1994), 353-369.

[B] J. Berge, The knots in $D^{2} \times S^{1}$ with nontrivial Dehn surgeries yielding $D^{2} \times S^{1}$, Topology Appl., 38 (1991), 1-19.

[BZ] S. Boyer and X. Zhang, Reducing Dehn filling and toroidal Dehn filling, Topology Appl., 68 (1996), 285-303.

[BZ2] _ On Culler-Shalen seminorms and Dehn filling, Ann. Math., 148 (1998), 737-801.

[CGLS] M. Culler, C.M. Gordon, J. Luecke and P. Shalen, Dehn surgery on knots, Ann. of Math., 125 (1987), 237-300. 
[D] J. Dean, Hyperbolic knots with small Seifert-fibered Dehn surgeries, PhD Thesis, University of Texas at Austin (1996); (available at http://www.math.lsa. umich.edu/dean/research).

[E] M. Eudave-Munoz, Band sum of links which yield composite links. The cabling conjecture for strongly invertible knots, Trans. Amer. Math. Soc., 330 (1992), 463-501.

[E2] _ On nonsimple 3-manifolds and 2-handle addition, Topology Appl., 55 (1994), 131-152.

[EW] M. Eudave-Munoz and Y. Wu, Nonhyperbolic Dehn fillings on hyperbolic 3manifolds, Pacific J. Math., 190 (1999), 261-275.

[Ga] D. Gabai, Foliations and the topology of 3-manifolds III, J. Diff. Geom., 26 (1987), 479-536.

[Ga2] - 1-bridge braids in solid tori, Topology Appl., 37 (1990), 221-235.

[GO] D. Gabai and U. Oertel, Essential laminations in 3-manifolds, Ann. Math., 130 (1989), 41-73.

[GS] F. Gonzalez-Acuna and H. Short, Knot surgery and primeness, Math. Proc. Camb. Phil. Soc., 99 (1986), 89-102.

[G] C. Gordon, Dehn surgery and satellite knots, Trans. Amer. Math. Soc., 275 (1983), 687-708.

[GL] C. Gordon and J. Luecke, Reducible manifolds and Dehn surgery, Topology, 35 (1996), 385-409.

[GL2] C. Gordon and J. Luecke, Only integral surgery can yield reducible manifolds, Math. Camb. Phil. Soc., 102 (1987), 94-101.

[GL3] Knots are determined by their complements, J. Amer. Math. Soc., 2 (1989), 371-415.

[GL4] unpublished.

[GL5] _ Toroidal and boundary-reducing Dehn fillings, Topology Appl., 93 (1999), 77-90.

[GW] C. Gordon and Y. Wu, Annular and boundary reducing Dehn fillings, Topology, to appear; (available at http://www.math.uiowa.edu/wu/papers/papers.html).

[GW2] _ Toroidal and annular Dehn fillings, Proc. London Math. Soc., 78 (1999), 662-700.

[GW3] _ Annular Dehn fillings, preprint; (available at http://www.math. uiowa.edu/wu/papers/papers.html).

[Ha] A. Hatcher, On the boundary curve of incompressible surfaces, Pacific J. Math., 99 (1982), 373-377.

[HS] C. Hayashi and K. Shimokawa, Symmetric knots satisfy the cabling conjecture, Math. Proc. Camb. Phil. Soc., 123 (1998), 501-529.

[H] J. Hempel, 3-Manifolds, Ann. Math. Studies, 86 (1976).

[Ho] J. Hoffman, There are no strict great $x$-cycles after a reducing or $P^{2}$ surgery on a knot, J. of Knots and Its Ramification, 7 (1998), 549-569.

[J] W. Jaco, Lectures on three-manifolds topology, CBMS Regional Conf. Ser. Math., 43 (1980). 
[KT] J. Kalliongis and C. Tsau, Seifert fibered surgery manifolds of composite knots, Proc. Amer. Math. Soc., 108 (1990), 1047-1053.

[Ma] D. Matignon, Dehn surgery on a knot with three bridges cannot yield $P^{3}$, Osaka J. Math., 34 (1997), 133-134.

[M] W. Menasco, Closed incompressible surfaces in alternating knot complements, Topology, 23 (1984), 37-44.

[MT] W. Menasco and M. Thistlethwaite, Surfaces with boundary in alternating knot exteriors, J. Reine Angew. Math., 426 (1992), 47-65.

[MM] K. Miyasaki and K. Motegi, Seifert fibered manifolds and Dehn surgery, Topology, 36 (1997), 579-603.

[MS] K. Morimoto and M. Sakuma, On unknotting tunnels for knots, Math. Ann., 289 (1991), 143-167.

[R] D. Rolfsen, Knots and Links, Publish or Perish Inc., 1976.

[Sch] M. Scharlemann, Producing reducible 3-manifolds by surgery on a knot, Topology, 29 (1990), 481-500.

[T] W. Thurston, Three dimensional manifolds, Kleinian groups and hyperbolic geometry, Bull. Amer. Math. Soc., 6 (1982), 357-381.

[W] Y. Wu, Dehn surgery on arborescent knots, J. Diff. Geo., 43 (1996), 171-197.

[W2] Sutured manifold hierarchies, essential laminations, and Dehn surgery, J. Diff. Geo., 48 (1998), 407-437.

[W3] , Incompressibility of surfaces in surgered 3-manifolds, Topology, 31 (1992), 271-279.

Received May 28, 1999. The first author was partially supported by NSF grant DMS 9626884. The second author was partially supported by NSF grant DMS 9971561.

Mathematics Department

SUNY AT BUFFALO

Buffalo, NY 14214-3093

E-mail address: menasco@math.buffalo.edu

Mathematics Department

SUNY AT BUFFALO

Buffalo, NY 14214-3093

E-mail address: xinzhang@math.buffalo.edu 